\title{
Step effects and coverage dependence of hydrogen atom diffusion on $\operatorname{Pt}(111)$ surfaces
}

\author{
C. Z. Zheng, C. K. Yeung, M. M. T. Loy, and Xudong Xiao* \\ Department of Physics, Hong Kong University of Science and Technology, Hong Kong, China \\ (Received 5 March 2004; revised manuscript received 14 June 2004; published 4 November 2004)
}

\begin{abstract}
Step effects on surface diffusion of hydrogen on stepped $\mathrm{Pt}(111)$ have been studied by linear optical diffraction technique over a temperature range from 90 to $150 \mathrm{~K}$. Diffusion anisotropy on stepped $\operatorname{Pt}(111)$ surfaces has been observed: the unexpected enhanced diffusion perpendicular to steps cannot be explained within the lattice gas model on stepped substrates, indicating a nonlocal and directional step effect. The coverage-dependent diffusion coefficient on flat $\mathrm{Pt}(111)$ surface was also measured over a wide coverage range from 0.1 to $0.8 \mathrm{ML}$. They were analyzed within the framework of the lattice gas model using quasichemical approximation, indicating that $\mathrm{H}-\mathrm{H}$ repulsive interaction can significantly affect the energy of saddle point as well as that at the adsorption sites.
\end{abstract}

DOI: 10.1103/PhysRevB.70.205402

PACS number(s): 68.43.Jk, 68.35.Dv, 68.35.Fx, 68.35.Ja

\section{INTRODUCTION}

The interaction of hydrogen with transition metal surfaces, in particular, platinum, has received extensive experimental ${ }^{1-17}$ and theoretical ${ }^{18-28}$ attention, primarily because platinum is an important heterogeneous catalyst in the hydrogenation reactions, and the hydrogen atom is the simplest chemisorption species which provides the ideal model system for testing the theoretical models and dynamical concepts. Hydrogen is also interesting due to its small mass that opens the possibility of observation of crossover from classical dynamics to quantum dynamics.

Despite extensive investigations the properties of $\mathrm{H} / \mathrm{Pt}(111)$ are still incompletely known and understood. For surface diffusion of $\mathrm{H} / \mathrm{Pt}(111)$, Graham et al. ${ }^{1}$ recently applied the quasielastic helium atom scattering (QHAS) technique and observed an Arrhenius temperature dependence with an activation energy and a prefactor of $68 \mathrm{meV}$ and $1.1 \times 10^{-3} \mathrm{~cm}^{2} / \mathrm{s}$, respectively, at a hydrogen coverage of $0.1 \mathrm{ML}$ in the temperature range $140-250 \mathrm{~K}$. An earlier measurement ${ }^{2}$ which used laser induced thermal desorption (LITD) technique in the temperature range 190-260 K reported much higher diffusion activation energies of $500-300 \mathrm{meV}$ for $0.001-0.33 \mathrm{ML}$ hydrogen coverage. Graham et al. ${ }^{1}$ had tentatively attributed the difference to a higher diffusion barrier in the vicinity of steps.

Images by scanning tunneling microscopy (STM) indicated that the heat shock ${ }^{29}$ from high power laser pulses in LITD technique could indeed have introduced a significant amount of surface defects such as steps, due to creation of stacking faults in the subsurface. For diffusion over a macroscopic scale over $10 \mu \mathrm{m}$, effects due to steps must be considered. Quantitative information on the steps such as their density and orientation in the original LITD experiment was unfortunately missing. It is generally believed that steps slow down diffusion in its perpendicular direction, as shown directly by photoemission electron microscopy for potassium on a $\operatorname{Pd}(111)$ surface $^{30,31}$ and by linearly optical diffraction method for $\mathrm{CO}$ on $\mathrm{Pt}(111) ;{ }^{32,33}$ yet $\mathrm{H}$ diffusion on stepped $\mathrm{W}(100)$ surface is hardly affected by the steps. ${ }^{34,35}$ Thus, experiment on well characterized stepped $\mathrm{Pt}(111)$ surfaces for $\mathrm{H}$ diffusion should be performed to quantitatively understand the influence of steps, and to experimentally test the tentative conclusion of Graham et al. ${ }^{1}$

In this paper, we present results of surface diffusion of $\mathrm{H}$ on stepped $\mathrm{Pt}(111)$ surfaces, using linear optical diffraction (LOD), on vicinal $\mathrm{Pt}(111)$ surface with specified miscuts. As shown for $\mathrm{CO} / \mathrm{Pt}(111),{ }^{32,33,36,37}$ the linear optical diffraction technique ${ }^{38-40}$ can be employed to study the step effects in both step-parallel and perpendicular directions. A number of advantages make the LOD technique particularly suited to study the step effects. First, with the LOD technique it is straightforward to extract the diffusion coefficient from the data without extensive modeling. Second, unlike the LITD method there is no need to use high-power laser pulses to repeatedly and completely remove the locally adsorbed $\mathrm{H}$, and avoid the possibility of introducing surface defects by heat shocks. Instead, a shallow ( $\sim 0.02 \mathrm{ML})$ one-dimensional hydrogen coverage grating is created by two interfering laser pulses, and the subsequent diffusion is monitored using a low power $\mathrm{cw}$ laser. Third, the LOD method has a large dynamic range, which allows diffusivity measurement from $10^{-15}$ to $10^{-7} \mathrm{~cm}^{2} / \mathrm{s}$, whereas, the QHAS technique is limited only to high diffusivities $\left(D \geqslant 5 \times 10^{-6} \mathrm{~cm}^{2} / \mathrm{s}\right)$. Last, since the period of the coverage grating is on the micron scale, many steps on a stepped surface are contained within each period, and measurement of effects due to steps can be isolated, particularly with different miscut samples with a varying step density in a controlled manner. By aligning the onedimensional adsorbate grating parallel or perpendicular to the steps, diffusion anisotropy can be measured.

Our results reveal very interesting step effects for $\mathrm{H}$ diffusion on $\mathrm{Pt}(111)$. While in the step-parallel direction the diffusion was affected by steps only to a small extent, $\mathrm{H}$ diffusion perpendicular to steps surprisingly becomes faster on $1^{\circ}$ and $2^{\circ}$ miscut samples. Slow-down of $\mathrm{H}$ diffusion perpendicular to steps was observed only for a high $\left(4^{\circ}\right)$ miscut surface. Our results on flat $\mathrm{Pt}(111)$ surface are in quantitative disagreement with the QHAS results. For completeness, we also include the coverage dependence of surface diffusion of $\mathrm{H}$ on flat $\mathrm{Pt}(111)$ over a coverage range from 0.1 to $0.8 \mathrm{ML}$ in this paper. 


\section{EXPERIMENT}

Five samples with different miscuts were used in the experiment: one flat (miscut $<0.1^{\circ}$ ) for terrace diffusion measurement, one each with $2^{\circ}$ or $4^{\circ}$ miscut off the (111) plane along [1-12] direction to provide A-type steps, and one each with $1^{\circ}$ or $2^{\circ}$ miscut off the (111) plane along [112] to provide B-type steps. The experiment was performed in an ultrahigh vacuum (UHV) chamber with a base pressure of $2.0 \times 10^{-10}$ Torr. Sample cleaning was performed by extensive cycles of $\mathrm{Ar}^{+}$sputtering, oxygen treatment at $1000 \mathrm{~K}$ and high temperature annealing at $1200 \mathrm{~K}$. The routine cleaning procedure of the $\mathrm{Pt}$ surface was to $\mathrm{Ar}^{+}$sputtering the surface at room temperature for $30 \mathrm{~min}$, followed by annealing at $1200 \mathrm{~K}$ for $5 \mathrm{~min}$. Cleanness and ordering of the sample were checked by Auger electron spectroscopy (AES) and low-energy electron diffraction. The sample temperature was controlled by electron beam heating and liquid-nitrogen cooling, and monitored by a $K$-type thermocouple spotwelded at the side of the samples.

We use the linear optical diffraction technique for surface diffusion measurement, which has been described in detail previously. ${ }^{38-40}$ Here, we summarize the key points. First, a uniform hydrogen layer with a prescribed coverage is deposited at $100 \mathrm{~K}$ on a flat or a stepped $\mathrm{Pt}(111)$ surface. Then a one-dimension grating of adsorbates is created at a diffusion temperature by interfering two laser beams (Nd: yttriumaluminum-garnet laser at $1.06 \mu \mathrm{m}, 10 \mathrm{~ns}$ ) at the surface via laser-induced thermal desorption. The depth of the grating is about $0.02 \mathrm{ML}$. The smearing of the adsorbate grating via surface diffusion can be detected by the decay of the firstorder linear diffraction signal of a $\mathrm{He}-\mathrm{Ne}$ laser beam with polarization modulation ${ }^{40}$

$$
S(t)=S(0) \exp (-t / \tau), \quad \text { with } \tau=s^{2} / 8 \pi^{2} D,
$$

where $D$ is the chemical diffusion coefficient and $s$ is the grating period. In the present study, $D$ was measured as a function of substrate temperature over a range 90-150 K.

In our experiment, the $\operatorname{Pt}(111)$ surface was first dosed with $\mathrm{H}_{2}$ gas at $\sim 100 \mathrm{~K}$ to the desired coverage by backfilling the chamber through a leak valve. For a flat $\operatorname{Pt}(111)$ sample, coverage was controlled by exposure whose corresponding coverage $\theta_{H}$ was evaluated from the thermal desorption spectra (TDS), ${ }^{12,13}$ with the absolute calibration performed at $1 \mathrm{ML}$. For stepped $\mathrm{Pt}(111)$ samples, coverage was set to $0.4 \mathrm{ML}$ by dosing the same amount of $\mathrm{H}_{2}$ gas as that on flat $\mathrm{Pt}(111)$ surface. ${ }^{41}$ The sample temperature was subsequently raised or lowered to the diffusion temperature before creating the $\mathrm{H}$ grating by laser induced thermal desorption. Once the grating was created, the first order diffraction signal from the grating was measured as a function of time as discussed earlier. We kept the diffusion temperature below the take-up desorption temperature to avoid the influence of thermal desorption on our diffusion measurements. Figure 1 shows typical data, from which the decay time $\tau$, and, knowing $s$, the diffusion coefficient $D$ can be readily obtained without the need for modeling.

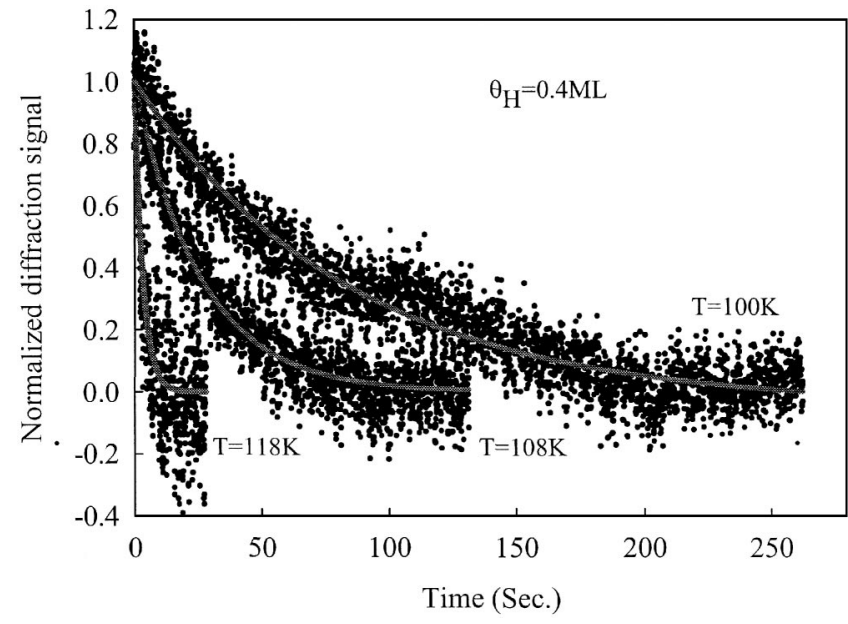

FIG. 1. Representative first-order diffraction signals vs time for $\mathrm{H}$ diffusion on a $<0.1^{\circ}$ miscut surface for $\theta_{\mathrm{H}}=0.4 \mathrm{ML}$ at temperatures of 118,108 , and $100 \mathrm{~K}$, respectively. The solid lines are single exponential fits. Data from the stepped surfaces are of similar quality.

\section{RESULTS AND DISCUSSION}

\section{A. Step effects on $\mathbf{H}$ diffusion on stepped $\operatorname{Pt}(111)$ surfaces}

To evaluate the step effects on surface diffusion, $\mathrm{H}$ diffusion on the stepped $\mathrm{Pt}(111)$ surface in the direction perpendicular (parallel) to steps was measured by aligning the coverage grating parallel (perpendicular) to steps. The experimental results at the coverage of $\theta_{H} \sim 0.4 \mathrm{ML}$ for B-type and A-type steps are shown in Figs. 2(a) and 2(b), respectively. For comparison, the diffusion coefficient on flat $\operatorname{Pt}(111)$ surface at the coverage of $\theta_{H} \sim 0.4 \mathrm{ML}$ is included. These values of surface diffusion coefficient were determined by fitting the decay of the first order linear diffraction signal as described earlier. The deduced diffusion activation energies and prefactors are given in Table I, with their error bars calculated from the standard errors of the corresponding fitting parameters. It is clear that on a given stepped surface diffusion perpendicular to steps $\left(D_{\perp}\right)$ is much faster than the diffusion parallel to steps $\left(D_{\|}\right)$for all the stepped surfaces except the one with $4^{\circ}$ miscut with A-type steps. Compared with the flat $\mathrm{Pt}(111)$ surface, diffusion in the direction parallel to steps is enhanced for all stepped surfaces. Thus, in contrast to the conventional view that steps hinder diffusion, a small density of steps $\left(1^{\circ}\right.$ or $2^{\circ}$ miscut in current study) enhances the diffusion rather than impedes the diffusion even perpendicular to steps. The diffusion perpendicular to steps is observed to speed up with step density for $1^{\circ}$ or $2^{\circ}$ miscut samples (both A and B type) and to drop for a $4^{\circ}$ miscut sample. (We have data only for A type steps, experiment on B type steps will be performed in the future to further check this point.)

We now discuss the step effects within the framework of lattice gas model on a stepped substrate. ${ }^{42}$ Independent whether the adsorbate-adsorbate interaction can be neglected or not, the total diffusion time over the surface in the stepperpendicular direction is the sum of diffusion time across terraces and diffusion time across steps. In spite of the de- 

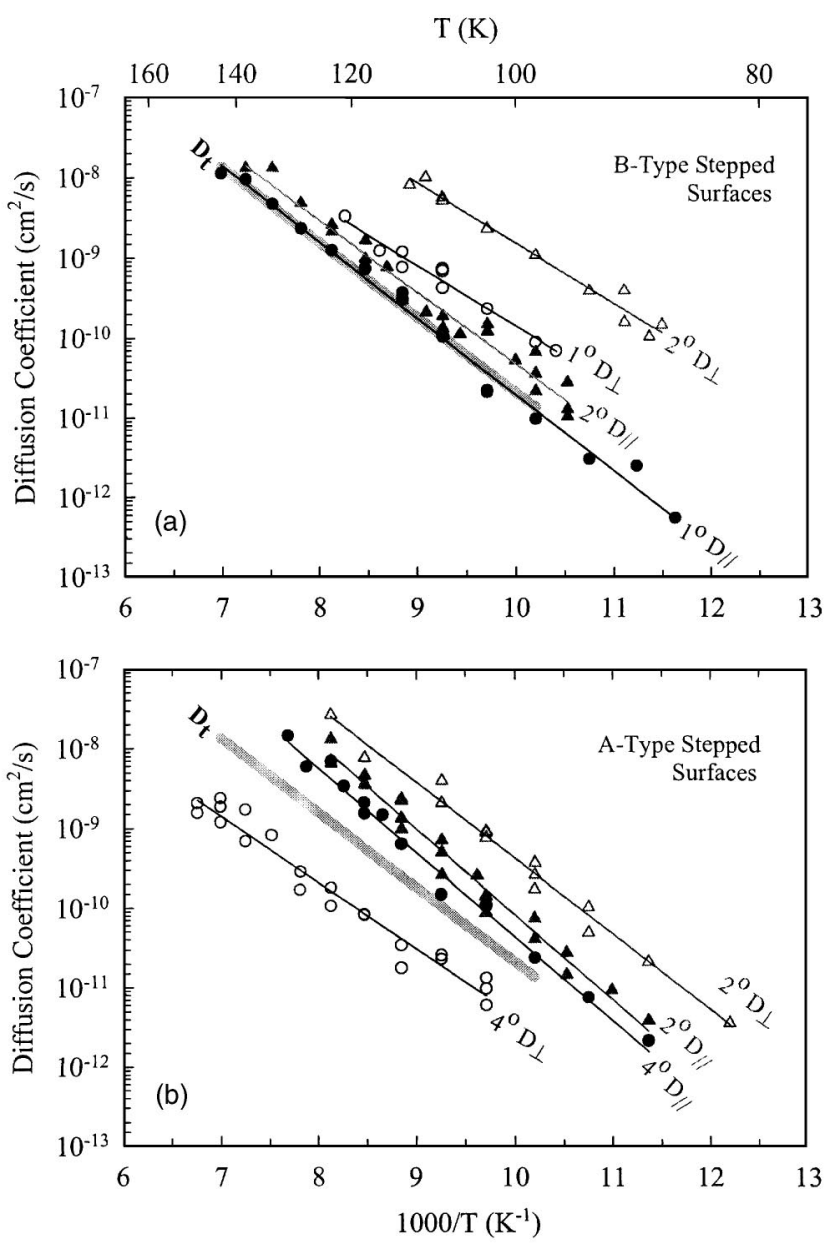

FIG. 2. Arrhenius plots of the diffusion coefficient $D$ for $H$ on (a) $1^{\circ}$ and $2^{\circ}$ B-type miscut and (b) $2^{\circ}$ and $4^{\circ}$ A-type miscut stepped $\mathrm{Pt}(111)$ surfaces at a coverage of $\theta_{H} \sim 0.4 \mathrm{ML}$.

tailed relation which is model dependent, the diffusion coefficient is inversely proportional to the diffusion time for a given system. The fact that most of the surface area on our stepped samples remains to be terrace would imply that the overall diffusion coefficient perpendicular to step $\left(D_{\perp}\right)$ must at most be comparable to terrace diffusion coefficient $\left(D_{t}\right)$, if not slower. Thus, one cannot account for the observed significant fast diffusion (about one to two orders of magnitude

TABLE I. Deduced diffusion activation energies and prefactors for $\mathrm{H}$ diffusion on stepped $\mathrm{Pt}(111)$ surfaces with miscut $1^{\circ}$ and $2^{\circ}$ for B-type step, and $2^{\circ}$ and $4^{\circ}$ for A-type step at the coverage of $\theta_{H} \sim 0.4$.

\begin{tabular}{lcccc}
\hline \hline & \multicolumn{2}{c}{ B-type step } & \multicolumn{2}{c}{ A-type step } \\
\multicolumn{1}{c}{$D_{\|}$} & $1^{\circ}$ & $2^{\circ}$ & $2^{\circ}$ & $4^{\circ}$ \\
\hline$E_{d}(\mathrm{meV})$ & $189 \pm 16$ & $177 \pm 17$ & $213 \pm 32$ & $209 \pm 17$ \\
$D_{0}\left(\mathrm{~cm}^{2} / \mathrm{s}\right)$ & $10^{-(1.2 \pm 0.3)}$ & $10^{-(1.3 \pm 0.3)}$ & $10^{0.67 \pm 0.36}$ & $10^{0.21 \pm 0.33}$ \\
$D_{\perp}$ & $1^{\circ}$ & $2^{\circ}$ & $2^{\circ}$ & $4^{\circ}$ \\
$E_{d}(\mathrm{meV})$ & $149 \pm 10$ & $148 \pm 12$ & $188 \pm 17$ & $158 \pm 19$ \\
$D_{0}\left(\mathrm{~cm}^{2} / \mathrm{s}\right)$ & $10^{-(2.3 \pm 0.5)}$ & $10^{-(1.3 \pm 0.4)}$ & $10^{0.13 \pm 0.36}$ & $10^{-(3.1 \pm 0.3)}$ \\
\hline \hline
\end{tabular}

faster than that on a flat surface) perpendicular to steps on vicinal $\mathrm{Pt}(111)$ surfaces for $1^{\circ}$ and $2^{\circ}$ miscut samples (both A and $B$ types) if the terrace diffusion is not affected by the steps.

To explain the faster diffusion perpendicular to steps than that on a flat surface, diffusion across the step edge must take a negligible amount of time compared to that over a terrace. More importantly, the diffusion speed over the terrace must be enhanced by the steps. Thus, the effect of steps must be nonlocal and extends to the terrace sites. While the definite mechanism has yet to be identified, we offer the following possibilities. First, steps may have a nonlocal effect that alters the potential energy surface or the dynamics for $\mathrm{H}$ on terraces some distance away from the steps. Second, a strong step-step interaction may exist. Since such interaction was not found for $\mathrm{CO}$ on these surfaces, adsorption of $\mathrm{H}$ atoms must participate in mediating this step-step interaction. Third, the lateral interaction among hydrogen atoms may have been significantly changed due to the presence of steps. $^{43}$ From contact potential measurement for $\mathrm{H}$ on stepped $\mathrm{Pt}(997)$ surface, ${ }^{13}$ it was found that $\mathrm{H}$ adsorbed on step site has a negative polarization while $\mathrm{H}$ adsorbed on terrace site has a positive polarization. These opposite polarizations of adsorbed $\mathrm{H}$ atom on a stepped surface will definitely alter the adsorbate-adsorbate interaction over a relatively long range and undermine the validity of a local diffusion model. All the earlier effects may manifest themselves in forms of nonuniform local $\mathrm{H}$ coverage as well as inhomogeneous diffusion dynamics. Last, it is possible that even at a low pressure $\left(\sim 10^{-10}\right.$ Torr), CO could still adsorb at steps from the residue gas of the UHV background as found during $\operatorname{Pt}(111)$ homoepitaxial growth. ${ }^{44}$ The adsorption of $\mathrm{CO}$ at steps might also affect $\mathrm{H}$ diffusion across the steps. At this stage, we cannot explain why $\mathrm{CO}$ could enhance $\mathrm{H}$ diffusion perpendicular to steps with a nonlocal effect. However, if $\mathrm{CO}$ did promote $\mathrm{H}$ diffusion perpendicular to steps, the disappearance of this enhancement effect on a surface with a higher step density $\left(4^{\circ} \mathrm{A}\right.$-type miscut) could be due to insufficient $\mathrm{CO}$ to saturate all the step sites.

Any nonlocal effect of the steps will also affect diffusion parallel to steps. The observed difference between the $\mathrm{H}$ diffusion parallel to steps and perpendicular to steps then would have to imply that the nonlocal step effect is also anisotropic, consistent with the one-dimensional nature of steps.

\section{B. Coverage dependence of $\mathrm{H}$ diffusion on flat $\mathrm{Pt}(111)$ surface}

In Fig. 3, the diffusion coefficients for various $\mathrm{H}$ coverages on a flat $\mathrm{Pt}(111)$ surface are depicted as a function of reciprocal temperature $1 / T$ in an Arrhenius plot. As expected for a flat surface, the results are independent of the directions of diffusion. Over a dynamic range of about two to three orders of magnitude, the measured diffusion coefficients can be well fitted by an Arrhenius relation, $D=D_{0} \exp \left(-E_{d} / k T\right)$. The deduced diffusion activation energies and prefactors for all the measured coverages are given in Table II. The activation energy values range about $5 \%-8 \%$ of the H-Pt interaction energy $(\sim 2.5 \mathrm{eV}),{ }^{12}$ typical for gas adsorbate diffusion on metal surfaces. ${ }^{45}$ As shown in Fig. 3, the coverage depen- 


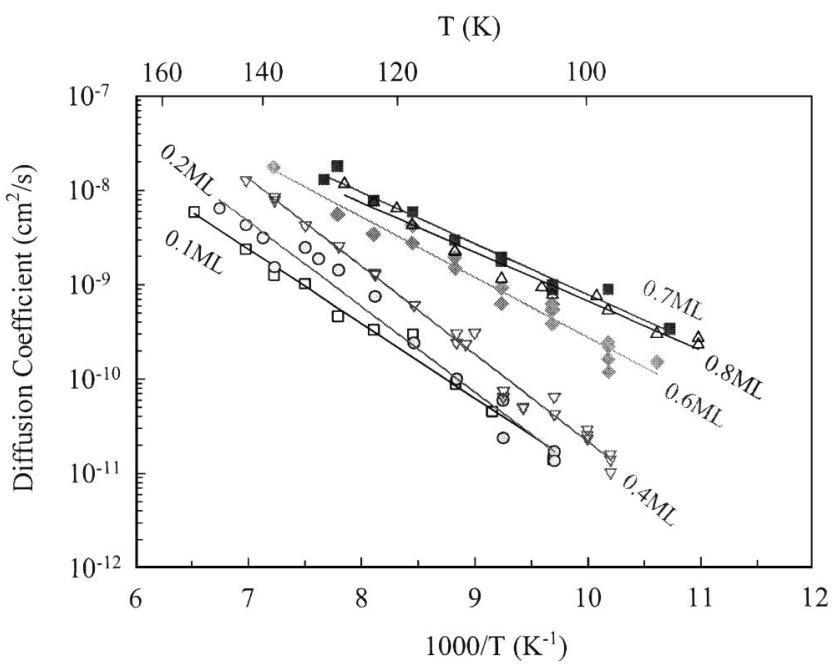

FIG. 3. Arrhenius plots of the diffusion coefficient $D$ for $\mathrm{H}$ on a flat $\mathrm{Pt}(111)$ surface over a coverage range from 0.1 to $0.8 \mathrm{ML}$ as indicated.

dence over the coverage ranges from 0.1 to $0.4 \mathrm{ML}$ and from 0.6 to $80 \mathrm{ML}$ is weak, but the dependence is significant from 0.4 to $0.6 \mathrm{ML}$.

The coverage dependent diffusion is caused by adsorbateadsorbate interaction. An analysis on the coverage dependence of activation energy and diffusion coefficient allows us to obtain some information about this lateral interaction for $\mathrm{H}$ on a flat $\mathrm{Pt}(111)$ surface. Figure 4 shows that the activation energy increases slightly at low hydrogen coverage and then decreases significantly at high hydrogen coverage. This behavior is qualitatively consistent with a $\mathrm{H}-\mathrm{H}$ repulsive interaction leading to a decreased effective barrier to surface diffusion at high coverage. ${ }^{45}$ Figure 5 also shows that the surface diffusion coefficient at five different temperatures increases monotonously with $\mathrm{H}$ coverage up to $\theta_{H}=0.7 \mathrm{ML}$ and slightly decreases at $\theta_{H}=0.8 \mathrm{ML}$. This behavior is consistent with a $\mathrm{H}-\mathrm{H}$ repulsive interaction, leading to a monotonously increasing diffusion coefficient with higher coverage until site blocking becomes important. ${ }^{45}$ The coverage dependence of the diffusion coefficient $D$ and activation energy $E_{d}$ can be modeled within the framework of the lattice gas model using the quasichemical approximation. ${ }^{46-49}$ In this model, only nearest neighbor lateral interaction is taken

TABLE II. Deduced diffusion activation energies and prefactors for $\mathrm{H}$ diffusion on a flat $\operatorname{Pt}(111)\left(<0.1^{0}\right.$ miscut $)$ surface for various coverages.

\begin{tabular}{ccc}
\hline \hline & $<0.1^{0}$ miscut Pt $(111)$ \\
$\theta(\mathrm{ML})$ & $E_{d}(\mathrm{meV})$ & $D_{0}\left(\mathrm{~cm}^{2} / \mathrm{s}\right)$ \\
\hline 0.1 & $157 \pm 17$ & $10^{-(3.1 \pm 0.3)}$ \\
0.2 & $179 \pm 18$ & $10^{-(2.0 \pm 0.3)}$ \\
0.4 & $185 \pm 15$ & $10^{-(1.3 \pm 0.2)}$ \\
0.6 & $127 \pm 16$ & $10^{-(3.1 \pm 0.3)}$ \\
0.7 & $108 \pm 10$ & $10^{-(3.7 \pm 0.2)}$ \\
0.8 & $104 \pm 10$ & $10^{-(3.9 \pm 0.2)}$ \\
\hline \hline
\end{tabular}

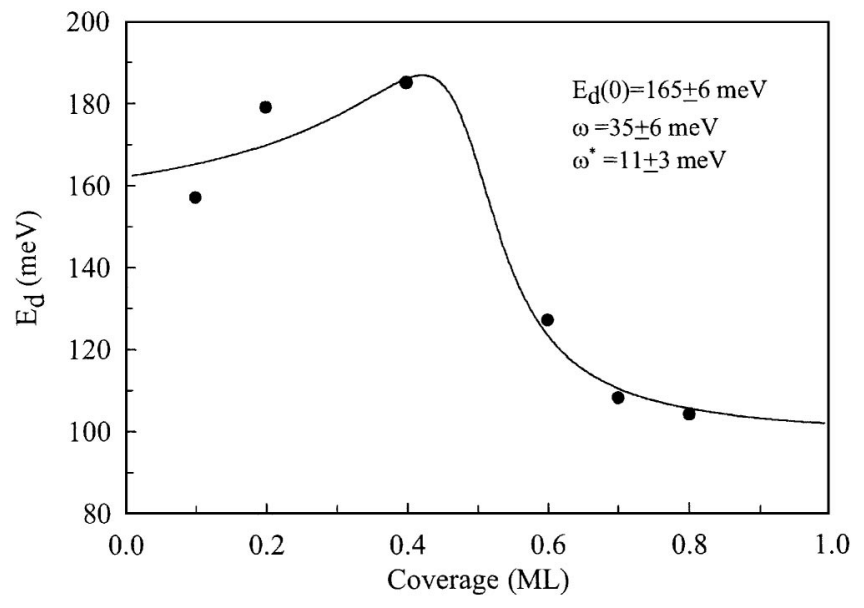

FIG. 4. Coverage dependence of diffusion activation energy $E_{d}$ for $\mathrm{H} / \mathrm{Pt}(111)$. The solid line represents a best fit based on the quasichemical approximation.

into account, and diffusion of adsorbed particles occurs via uncorrelated activated jumps to the nearest neighbor empty sites. The diffusion coefficient is given by ${ }^{49}$

$$
D(\theta)=S \frac{\partial \exp (\mu / k T)}{\partial \theta} D(0)
$$

with

$$
\begin{gathered}
S=P_{O O}\left[\frac{P_{O O}+1 / 2 P_{A O} \exp \left(-\omega^{*} / k T\right)}{P_{O O}+1 / 2 P_{A O}}\right]^{2 z-2}, \\
\exp (\mu / k T)=\frac{\theta}{1-\theta}\left[\frac{P_{A A} \exp (\omega / k T)+1 / 2 P_{A O}}{P_{A A}+1 / 2 P_{A O}}\right]^{z},
\end{gathered}
$$

where $z$ is the coordination number of the lattice which equals 6 in our case; $\mu$ is the chemical potential of adsorbed particles; $P_{O O}, P_{A O}$, and $P_{A A}$ are the quasichemical probability that two adjacent sites are occupied by zero, one, and two particles, respectively. They can be obtained by solving the combination of the quasichemical equation $P_{A A} P_{O O}$

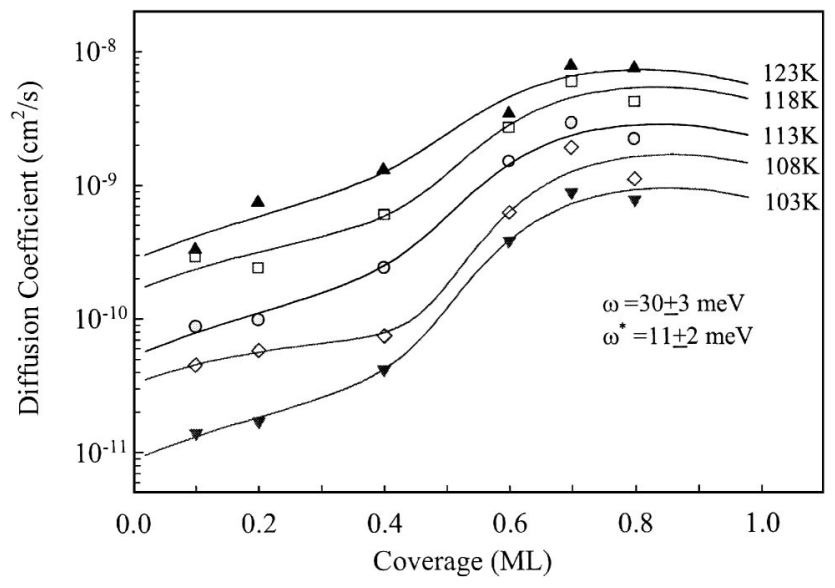

FIG. 5. Coverage dependence of surface diffusion coefficient $D$ of $\mathrm{H} / \mathrm{Pt}(111)$ at five different temperatures. The solid lines represent best fits based on the quasichemical approximation. 
$=\frac{1}{4} P_{A O} \exp (-\omega / k T)$ and two balance equations $P_{A A}+P_{A O}$ $+P_{O O}=1$ and $2 P_{A A}+P_{A O}=2 \theta$; the two parameters $\omega$ and $\omega^{*}$ are used to model the influence of $\mathrm{H}-\mathrm{H}$ interactions on the surface diffusion. $\omega$ is the interaction energy between every pair of adsorbed particles with other nearby sites empty, and $\omega^{*}$ the interaction energy between the saddle point complex and the nearest neighbor adsorbed particle. For each additional particle placed at any of the six nearest neighbor sites surrounding the adsorbate, the energy of the adsorbate is increased by an amount of $\omega$, and for each additional particle placed at any of the ten nearest neighbor sites surrounding the adsorbate and the empty site that the adsorbate will jump into, the energy of the saddle point is increased by an amount $\omega^{*}$. The diffusion activation energy is given by $E_{d}(T, \theta)$ $=[\partial \ln D(\theta, T) / \partial(1 / k T)]$, where the effective temperature for activation energy is set equal to $110 \mathrm{~K}$ in our case.

The solid lines in Fig. 5 show the best fits of the surface diffusion coefficient which yield parameters $\omega=30 \pm 3 \mathrm{meV}$, and $\omega^{*}=11 \pm 2 \mathrm{meV}$. This is consistent with the fitting result of the activation energy with $E_{d}=165 \pm 6 \mathrm{meV}$ at coverage $\theta_{H} \rightarrow 0, \omega=35 \pm 6 \mathrm{meV}$, and $\omega^{*}=11 \pm 3 \mathrm{meV}$ as shown in Fig. 4. The obtained $\omega$ value is about three times larger than the value of $7.8 \mathrm{meV}$ deduced by Graham et al. ${ }^{1}$ using the same quasichemical approximation to analyze their QHAS experimental data. The difference is that they have set $\omega^{*}$ $=0$ in addition to the experimentally obtained different diffusion activation energies. Our results are consistent with the TDS and contact potential measurements, ${ }^{12,15}$ from which the coverage dependent desorption energy leads to an estimate of $\omega-\omega_{d}^{*}\left(\omega_{d}^{*}\right.$ is the H-H interaction energy at the saddle point for desorption) to be in the range from 15 to $25 \mathrm{meV}$.

\section{Comparison with previous results}

We compare the magnitude of our diffusion activation energy at low $\mathrm{H}$ coverage $\theta_{H}=0.1 \mathrm{ML}$ with previous results. Our value of $\sim 157 \mathrm{meV}$ is close to the $194 \mathrm{meV}$ estimated from field emitter measurement; ${ }^{3}$ but in between the $\sim 500 \mathrm{meV}$ measured by LITD $^{2}$ and the $\sim 68 \mathrm{meV}$ measured by QHAS. ${ }^{1}$ The range of the calculated activation energy from theoretical models is likewise rather broad, ranging from 200 to $\sim 80 \mathrm{meV} \cdot{ }^{19,26-28}$

The difference in the measured diffusion activation energy by LOD and by QHAS may come from the different diffusion length scale involved in these techniques. ${ }^{50}$ Due to the macroscopic diffusion length scale of $\sim 5 \mu \mathrm{m}$ used in LOD, the effect of surface steps on $\mathrm{H}$ diffusion is unavoidable. It would be reasonable to attribute ${ }^{1,50}$ the difference in the reported activation energy to the possibly higher energy barrier for diffusion across steps. Indeed, the present study is to put this supposition to a quantitative experimental test by direct measurement of diffusion parallel to and perpendicular to steps: for this to hold, the energy barrier for crossing the steps would have to be much higher than that on terrace. Our results, shown in Fig. 2 and Table I, show conclusively that for the case of $\mathrm{H}$ on $\mathrm{Pt}(111)$ the energy barrier for motion across steps is not substantially higher. Thus, steps cannot be the cause for the difference in the reported values of activation energy.
$\mathrm{T}(\mathrm{K})$

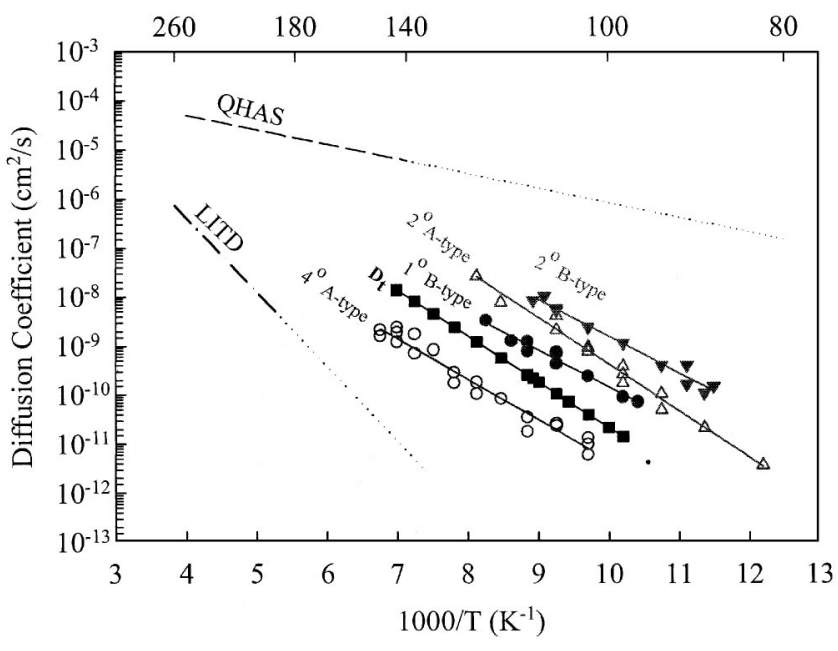

FIG. 6. Comparison of $\mathrm{H}$ diffusion results on $\mathrm{Pt}(111)$. Diffusion results from LITD at $\theta_{H} \sim 0.33 \mathrm{ML}$ and QHAS at $\theta_{H} \sim 0.4 \mathrm{ML}$ are indicated by dash-dot line, and their extrapolations are indicated by dotted lines. Diffusion data from the present study at $\theta_{H} \sim 0.4 \mathrm{ML}$ on $<0.1^{\circ}$ miscut surfaces, $2^{\circ}, 4^{\circ}$ miscut surfaces with A-type steps, and $1^{\circ}, 2^{\circ}$ miscut surfaces with B-type steps in step-perpendicular direction are all replotted and labeled.

Impurities or other point defects are the other candidates that may hinder surface diffusion of adsorbates. ${ }^{51-54}$ We note that surface mobility of hydrogen can decrease by a factor of $\sim 60$ as the surface carbon coverage was increased from $\theta_{C}$ $=0$ to $\theta_{C}=0.42 \mathrm{ML}$ at $T=300 \mathrm{~K} .{ }^{52}$ However, in our case, the amount of impurities was below the AES sensitivity. Comparing our measured diffusion coefficient $D$ with the extrapolated value from QHAS results, we see a difference of more than four orders of magnitude (see Fig. 6), which is difficult to attribute to impurities below AES sensitivity. As for point defects, with the samples well annealed at high temperature, the concentration of point defects is not expected to be high. If these defects are trapping centers, a small amount of $\mathrm{H}$ atoms may passivate them and the observed $\mathrm{H}$ diffusion in our experiment should be the property of those adsorbed on regular terrace sites. Thus the presence of point defects is not likely to account for the difference.

Barth $^{50}$ has provided an excellent review on the general subject of transport of adsorbates at metal surfaces using various techniques including field ion microscopy, STM, LITD, QHAS, and LOD. The different techniques, probing different physical quantities from which the diffusion coefficient $D$ can be deduced, give information on length scales from $10 \AA$ to $1000 \mu \mathrm{m}$, covering a range of values for $D$ from $10^{-16}$ to $10^{-5} \mathrm{~cm}^{2} / \mathrm{s}$ : each with its optimal range and none can cover the entire range. In particular, LOD has been shown to have excellent dynamic range as well as straightforward deduction of the diffusion coefficient. Of course, it is essentially a macroscopic technique and possible effects from steps and defects must be addressed appropriately. The QHAS technique has the potential to provide truly microscopic scale information on the motion of the diffusing atoms. But there are a number of practical challenges to overcome: First, this information is encoded into a broadened 
linewidth, $\Gamma$, of the "quasielastic" peak in reciprocal space, and the broadening due to the diffusion process can be recovered by first subtraction from the background, and then deconvoluted from the instrument response. This instrumental response, being about $300 \mu \mathrm{V}$, limits the technique to fast processes with $D>10^{-6} \mathrm{~cm}^{2} / \mathrm{s} .{ }^{55}$ Second, this deconvoluted $\Gamma$ is directly related to $D$ through the expression $\Gamma$ $=2 \hbar D(\Delta K)^{2}(\Delta K$ is the momentum transfer) only in the limit $\Delta K \rightarrow 0$. Ge and $\mathrm{King}^{56}$ showed that in general it is by no means straightforward to extract diffusion parameters from QHAS linewidth measurement because this extraction process is clearly "model dependent." Recently, Jardine et al. ${ }^{57}$ provided a detailed review and critical analysis of QHAS experiments and interpretations, pointing out the need for molecular dynamics (MD) simulation to generate a correct interpretation of QHAS results. Finally, the present instrumental limit in energy resolution discussed earlier severely limits the dynamic range of QHAS: for $\mathrm{H}$ on $\mathrm{Pt}(111)$, this was only about one order of magnitude (e.g., Fig. 11 in Ref. 1 ), with the lower limit of the deduced $D$ being close to the detection limit of $10^{-6} \mathrm{~cm}^{2} / \mathrm{s}$. (This assumes the capability to reliably obtain a deconvoluted width of only $1 / 100$ of the instrument width. Barth $^{50}$ placed the detection limit to be 5 $\times 10^{-6} \mathrm{~cm}^{2} / \mathrm{s}$.) The diffusion parameters reported in Ref. 1 should therefore be quoted with caution. In particular, it would be highly desirable that a MD simulation be performed for this case, similar to that in Jardine et al., ${ }^{57}$ to provide a further check on the reported diffusion parameters in Ref. 1. It will be important to see whether future QHAS experiments with improved resolution ${ }^{57}$ give the same higher value of $D$ and lower value of $E_{d}$ for the present $\mathrm{H}$ on $\operatorname{Pt}(111)$ system, and in many other cases when comparisons from other techniques are available. ${ }^{36,58-65}$

We also note the different temperature range used in the two experiments. As shown by TDS of $\mathrm{H} / \mathrm{Pt}(111),{ }^{12}$ the take-up desorption temperature of $\mathrm{H}$ is about $170 \mathrm{~K}$ at high coverage and $200 \mathrm{~K}$ at low coverage, thus, the $\mathrm{H}$ atoms will partially desorb from the $\operatorname{Pt}(111)$ over the QHAS measurement temperature range from 140 to $250 \mathrm{~K}$. The partial desorption of $\mathrm{H}$ will not only affect the coverage of $\mathrm{H}$, but also the $\mathrm{H}-\mathrm{H}$ lateral interaction. In our LOD measurement, the coverage dependence was measured over the temperature range from 90 to $150 \mathrm{~K}$, which is always below the take-up desorption temperature and assures that the same coverage was used in the diffusion measurements.

Our value for the activation energy is substantially less than the 300-500 meV value from the LITD method. Based on our observation that diffusion slows down only for high step densities in our $4^{\circ}$ miscut sample, the step densities in the LITD experiment would have to be extremely high to account for their data. A more likely scenario is the presence of high densities of point defects, generated by the large number of high energy laser pulses required in the LITD experiment. These defects will act as traps for $\mathrm{H}$ atoms, and particularly for very low $\left(10^{-3} \mathrm{ML}\right)$ coverage in the LITD experiment, these trapped $\mathrm{H}$ will contribute significantly to the measured diffusion, leading to high values of activation energy.

\section{CONCLUSION}

In conclusion, with the linear optical diffraction method we have systematically studied the step effects on $\mathrm{H}$ diffusion on stepped $\mathrm{Pt}(111)$, using a number of miscut samples. On the $1^{\circ}, 2^{\circ}$ B-type miscut, and $2^{\circ}$ A-type miscut samples, surprisingly, diffusion perpendicular to steps was not impeded but rather enhanced compared to flat surface results. Only on the high step density sample with $4^{\circ}$ A-type miscut a suppression of diffusion speed was measured. Diffusion parallel to steps except for $4^{\circ}$ A-type miscut samples were found slower than that perpendicular to steps. Our observation cannot be explained within the framework of lattice gas model and must require a non-local step effect, as opposed to previous studies. Such a nonlocal effect remains as a topic for future theoretical investigation.

We have also measured the coverage dependent $\mathrm{H}$ diffusion on flat $\mathrm{Pt}(111)$ over a wide temperature range from 90 to $150 \mathrm{~K}$ and over a wide range of coverage from 0.1 to 0.8 ML. The activation energy increases slightly from 157 to $185 \mathrm{meV}$ for $\theta=0.1$ to $0.4 \mathrm{ML}$ and drops from 127 to $104 \mathrm{meV}$ for $\theta=0.6$ to $0.8 \mathrm{ML}$. The responsible repulsive $\mathrm{H}-\mathrm{H}$ interaction has been analyzed within quasi-chemical approximation, and we found that the $\mathrm{H}-\mathrm{H}$ interaction affects the adsorbed $\mathrm{H}$ atom energy at adsorption site as well as at the saddle point.

\section{ACKNOWLEDGMENTS}

The authors thank the help from Dr. Lei Cai at the initial stage of the experiment and wish to acknowledge financial support from the Research Grant Council of Hong Kong through Grant No. HKUST6147/99P, the HKUST RIG Grant, and the William Mong Solid State Cluster Laboratory.

\footnotetext{
*Author to whom correspondence should be addressed. Email address: phxudong@ust.hk

${ }^{1}$ A. P. Graham, A. Menzel, and J. P. Toennies, J. Chem. Phys. 111, 1676 (1999).

${ }^{2}$ E. G. Seebauer and L. D. Schmidt, Chem. Phys. Lett. 123, 129 (1986)

${ }^{3}$ R. Lewis and R. Gomer, Surf. Sci. 17, 333 (1969).

${ }^{4}$ K. Umezawa, T. Ito, M. Asada, S. Nakanishi, P. Ding, W. A.
}

Lanford, and B. Hjörvarsson, Surf. Sci. 387, 320 (1997).

${ }^{5}$ B. J. J. Koeleman, S. T. de Zwart, A. L. Boers, B. Poelsema, and L. K. Verheij, Phys. Rev. Lett. 56, 1152 (1986).

${ }^{6}$ K. M. Lui, Y. Kim, W. M. Lau, and J. W. Rabalais, J. Appl. Phys. 86, 5256 (1999).

${ }^{7}$ A. M. Baro and H. IbacH, Surf. Sci. 92, 237 (1980).

${ }^{8}$ B. J. J. Koeleman, S. T. de Zwart, A. L. Boers, B. Poelsema, and L. K. Verhey, Nucl. Instrum. Methods Phys. Res. 218, 225 
(1983).

${ }^{9}$ A. T. Gee, B. E. Hayden, C. Mormiche, and T. S. Nunney, J. Chem. Phys. 112, 7660 (2000).

${ }^{10}$ B. Poelsema, L. K. Verheij, and G. Comsa, Surf. Sci. 152/153, 496 (1985).

${ }^{11}$ K. Christmann, Surf. Sci. Rep. 9, 1 (1988).

${ }^{12}$ K. Christmann, G. Ertl, and T. Pignet, Surf. Sci. 54, 365 (1976).

${ }^{13}$ K. Christmann and G. Ertl, Surf. Sci. 60, 365 (1976).

${ }^{14}$ H. Wang, R. G. Tobin, D. K. Lambert, G. B. Fisher, and C. L. DiMaggio, Surf. Sci. 330, 173 (1995).

${ }^{15}$ R. W. McCabe and L. D. Schmidt, Surf. Sci. 65, 189 (1977).

${ }^{16}$ K. Fukutani, A. Itoh, M. Wilde, and M. Matsumoto, Phys. Rev. Lett. 88, 116101 (2002).

${ }^{17}$ L. J. Richter and W. Ho, Phys. Rev. B 36, 9797 (1987).

${ }^{18}$ S. C. Badescu, K. Jacobi, Y. Wang, K. Bedürftig, G. Ertl, P. Salo, T. Ala-Nissila, and S. C. Ying, Phys. Rev. B 68, 205401 (2003).

${ }^{19}$ S. C. Badescu, P. Salo, T. Ala-Nissila, S. C. Ying, K. Jacobi, Y. Wang, K. Bedurftig, and G. Ertl, Phys. Rev. Lett. 88, 136101 (2002).

${ }^{20}$ K. Nobuhara, H. Kasai, H. Nakanishi, and A. Okiji, Surf. Sci. 507-510, 82 (2002).

${ }^{21}$ H. P. Kaukonen and R. M. Nieminen, Surf. Sci. 247, 43 (1991).

${ }^{22}$ K. Nobuhara, H. Nakanishi, H. Kasai, and A. Okiji, J. Appl. Phys. 91, 1855 (2002).

${ }^{23}$ R. A. Olsen, G. J. Kroes, and E. J. Baerends, J. Chem. Phys. 111, 11155 (1999).

${ }^{24}$ K. Nobuhara, H. Nakanishi, H. Kasai, and A. Okiji, J. Appl. Phys. 88, 6897 (2000).

${ }^{25}$ G. Papoian, J. K. Norskov, and R. Hoffmann, J. Am. Chem. Soc. 122, 4129 (2000).

${ }^{26}$ G. Kallen and G. Wahnstrom, Phys. Rev. B 65, 033406 (2001).

${ }^{27}$ P. J. Feibelman and D. R. Hamann, Surf. Sci. 182, 411 (1987).

${ }^{28}$ P. Blandin and P. Ballone, Surf. Sci. 331-333, 891 (1995).

${ }^{29}$ J. Frohn, J. Reynolds, and T. Engel, Surf. Sci. 320, 93 (1994).

${ }^{30}$ M. Ondrejcek, W. Stenzel, H. Conrad, V. Chab, Z. Chvoj, W. Engel, and A. M. Bradshaw, Chem. Phys. Lett. 215, 528 (1993).

${ }^{31}$ M. Ondrejcek, V. Chab, W. Stenzel, M. Snabl, H. Conrad, and A. M. Bradshaw, Surf. Sci. 331-333, 764 (1995).

${ }^{32}$ J. Ma, L. Cai, X. D. Xiao, and M. M. T. Loy, Surf. Sci. 425, 131 (1999).

${ }^{33}$ J. Ma, X. D. Xiao, and M. M. T. Loy, Surf. Sci. 436, L661 (1999).

${ }^{34}$ L. Cai, C. Z. Zheng, K. L. Man, M. S. Altman, E. Granato, T. Ala-Nissila, S. C. Ying, and X. D. Xiao, Phys. Rev. B 68, 075422 (2003).

${ }^{35}$ L. Cai, M. S. Altman, E. Granato, T. Ala-Nissila, S. C. Ying, and X. D. Xiao, Phys. Rev. Lett. 88, 226105 (2002).

${ }^{36}$ J. Ma, X. D. Xiao, J. J. DiNardo, and M. M. T. Loy, Phys. Rev. B 58, 4977 (1998)

${ }^{37}$ J. Ma, X. D. Xiao, and M. M. T. Loy, Surf. Sci. 423, 85 (1999).

${ }^{38}$ X. D. Xiao, X. D. Zhu, W. Daum, and Y. R. Shen, Phys. Rev. Lett. 66, 2352 (1991).

${ }^{39}$ X. D. Xiao, X. D. Zhu, W. Daum, and Y. R. Shen, Phys. Rev. B 46, 9732 (1992).

${ }^{40}$ X. D. Xiao, Y. Xie, and Y. R. Shen, Surf. Sci. 271, 295 (1992).
${ }^{41}$ In our experiment, the same exposure of $\mathrm{H}_{2}(6 L)$ was used for stepped surfaces with different step densities. Based on the TDS results from a higher density stepped surface, $\operatorname{Pt}(997)\left(\sim 6.5^{\circ}\right.$ miscut) on which dosing $6 \mathrm{~L}$ leads to a coverage of $\sim 0.1 \mathrm{ML}$ higher than that on the flat surface $(\sim 0.4 \mathrm{ML})$ at the same exposure, we estimated that the $\mathrm{H}$ coverage on $1^{\circ}, 2^{\circ}$, and $4^{\circ}$ surfaces to the $\sim 0.41,0.43$, and $0.46 \mathrm{ML}$, respectively. From Fig. 3, diffusion coefficient due to the coverage difference alone on the $1^{\circ}$ and $2^{\circ}$ miscut samples would at most increase by a factor of $\sim 3$, much smaller than the observed step effect with one to two orders of magnitude enhancement. Thus, we can safely neglect the coverage difference in our discussion. Furthermore, the coverage effect alone should cause the same enhancement for diffusion perpendicular to steps and diffusion parallel to steps.

${ }^{42}$ J. Merikoski and S. C. Ying, Phys. Rev. B 56, 2166 (1997).

${ }^{43}$ A. V. Myshlyavtsev and V. P. Zhdanov, Surf. Sci. 291, 145 (1993).

${ }^{44}$ M. Kalff, G. Comsa, and T. Michely, Phys. Rev. Lett. 81, 1255 (1998).

${ }^{45}$ R. Gomer, Rep. Prog. Phys. 53, 917 (1990).

${ }^{46}$ V. P. Zhdanov, Surf. Sci. 149, L13 (1985).

${ }^{47}$ D. A. Reed and G. Ehrlich, Surf. Sci. 102, 588 (1981).

${ }^{48}$ R. Fowler and D. A. Guggenheim, Statistical Thermodynamics (Cambridge University Press, Cambridge, 1949), Chap. 10.

${ }^{49}$ V. P. Zhdanov, Elementary Physicochemical processes on Solid Surfaces (Plenum Press, New York, 1991), p. 272.

${ }^{50}$ J. V. Barth, Surf. Sci. Rep. 40, 75 (2000).

${ }^{51}$ J. L. Brand, A. A. Deckert, and S. M. George, Surf. Sci. 194, 457 (1988).

${ }^{52}$ C. H. Mak, B. G. Koehler, J. L. Brand, and S. M. George, J. Chem. Phys. 87, 2340 (1987).

${ }^{53}$ X. D. Xiao, Y. Xie, C. Jakobsen, H. Galloway, M. Salmeron, and Y. R. Shen, Phys. Rev. Lett. 74, 3860 (1995).

${ }^{54}$ X. D. Xiao, Y. Xie, C. Jakobsen, and Y. R. Shen, Phys. Rev. B 56, 12529 (1997).

${ }^{55}$ J. Ellis, A. P. Graham, F. Hofmann, and J. P. Toennies, Phys. Rev. B 63, 195408 (2001).

${ }^{56}$ Q. Ge and D. A. King, J. Chem. Phys. 114, 1053 (2001).

${ }^{57}$ A. P. Jardine, J. Ellis, and W. Allison, J. Phys.: Condens. Matter 14, 6173 (2002).

${ }^{58}$ A. P. Graham and J. P. Toennies, Europhys. Lett. 42, 449 (1998).

${ }^{59}$ M. F. Bertino, F. Hofmann, W. Steinhogl, and J. P. Toennies, J. Chem. Phys. 105, 11297 (1996).

${ }^{60}$ J. Ellis, A. P. Graham, and J. P. Toennies, Phys. Rev. Lett. 82, 5072 (1999).

${ }^{61}$ H. Froitzheim and M. Schulze, Surf. Sci. 320, 85 (1994).

${ }^{62}$ V. J. Kwasniewski and L. D. Schmidt, Surf. Sci. 274, 329 (1992).

${ }^{63}$ M. Croci, C. Félix, G. Vandoni, W. Harbich, and R. Monot, Surf. Sci. 290, L667 (1992).

${ }^{64}$ X. D. Xiao, Y. Xie, and Y. R. Shen, Phys. Rev. B 48, 17452 (1993).

${ }^{65}$ D. L. Meixner and S. M. George, J. Chem. Phys. 98, 9115 (1993). 